\title{
As bitter as a trombone: Synesthetic correspondences in nonsynesthetes between tastes/flavors and musical notes
}

\author{
Anne-Sylvie Crisinel and Charles Spence \\ University of Oxford, Oxford, England
}

\begin{abstract}
In parallel to studies of various cases of synesthesia, many cross-modal correspondences have also been documented in nonsynesthetes. Among these correspondences, implicit associations between taste and pitch have been reported recently (Crisinel \& Spence, 2009, 2010). Here, we replicate and extend these findings through explicit matching of sounds of varying pitch to a range of tastes/flavors. In addition, participants in the experiment reported here also chose the type of musical instrument most appropriate for each taste/flavor. The association of sweet and sour tastes to high-pitched notes was confirmed. By contrast, umami and bitter tastes were preferentially matched to low-pitched notes. Flavors did not display such strong pitch associations. The choice of musical instrument seems to have been driven primarily by a matching of the hedonic value and familiarity of the two types of stimuli. Our results raise important questions about our representation of tastes and flavors and could also lead to applications in the marketing of food products.
\end{abstract}

Synesthesia is an intriguing condition. Although the first detailed scientific report on synesthesia dates from more than a century ago (Galton, 1880), its mechanisms are still unclear (Cytowic \& Eagleman, 2009). More attention has been devoted by researchers recently to various cases of synesthesia (see Hochel \& Milán, 2008, for a review; see also Harrison, 2001). But are the perceptual experiences of synesthetes so very different from those of nonsynesthetes? Our senses certainly do not work in isolation from each other. We live in a multisensory world, and our brains constantly combine information from different sensory modalities in order to make sense of our environment (see Calvert, Spence, \& Stein, 2004). The senses of taste and smell are so tightly combined in the evaluation of flavor that it is sometimes considered a form of synesthesia that is common to us all (Auvray \& Spence, 2008; Small \& Prescott, 2005; Stevenson \& Tomiczek, 2007; see also Djordjevic, Zatorre, \& Jones-Gotman, 2004). Moreover, the evaluation of the sweetness (i.e., a gustatory property) of a novel odor can be modified simply by pairing it during training with a sweet taste (Stevenson, Boakes, \& Prescott, 1998). A number of other cross-modal associations have now also been reported, such as between pitch and visual size (Evans \& Treisman, 2010; Gallace \& Spence, 2006; Parise \& Spence, 2009), between brightness and the frequency of vibrotactile stimuli (Martino \& Marks, 2000), or between colors and tastes (O'Mahony, 1983). The use of audiovisual metaphors for loudness, pitch, and brightness has also been reported (Marks, 1982). These associations are different from those present in synesthetes in that they are bidirectional (synesthesia is usually thought of as being unidirectional, but see Johnson, Jepma, \& de Jong, 2007), and a stimulus presented in one sensory modality does not elicit a conscious experience in another modality. However, the existence of these cross-modal associations supports the hypothesis that synesthesia might originate in feedback connections from a point of convergence of the two sensory pathways (Grossenbacher \& Lovelace, 2001). Several researchers have argued in recent years that cross-modal associations and synesthesia may be usefully compared in an effort to better understand both phenomena (e.g., Sagiv \& Ward, 2006; Ward, Huckstep, \& Tsakanikos, 2006).

Associations between tastes and particular pitches have been reported previously by Holt-Hansen $(1968,1976)$ in a comparison of different brands of beer. It has also recently been shown that basic tastes are associated to relative pitch (Crisinel \& Spence, 2009, 2010): In implicit association tasks, participants tend to associate sweet and sour tastes with high-pitched sounds. In these studies, tastes were evoked via the names of food or drink items. This method could therefore not totally exclude the possibility that it was the names themselves that may have had an influence on the associations observed (see also Simner, Cuskley, \& Kirby, 2010). Moreover, this method introduced variability between participants depending on their experience with the food/drink items that were used. For example, coffee, which was intended to represent a bitter taste, might have evoked a sweet taste in those participants who normally drank their coffee with sugar and milk.

Our goal in the present study was to replicate Crisinel and Spence's $(2009,2010)$ recent studies but using real tastants and flavors instead of merely the names of such

A.-S. Crisinel, anne-sylvie.crisinel@psy.ox.ac.uk 
items. We also greatly extended the range of stimuli that we explored: The tastants included various flavors on top of the five basic tastes, ${ }^{1}$ and the sounds used varied in the types of instrument used to play them and in their pitch. The participants in the present study had to explicitly match a sound to each of a series of tastes/flavors.

\section{METHOD}

\section{Participants}

Thirty-four participants took part in the experiment (20 female, $20-37$ years old). The participants reported no cold or other impairment of their senses of smell and taste and no hearing impairment. They were instructed not to have any meal, coffee, or to smoke in the 30 min prior to taking part in the study. The experiment lasted for approximately $40 \mathrm{~min}$, and the participants were compensated for their time by a $£ 5$ (UK Sterling) gift voucher. A subset of 17 participants (12 female, 20-34 years old) also took part in the second session.

\section{Stimuli}

The 12 gustatory stimuli were chosen to represent the five basic tastes (caffeine, bitter; citric acid, sour; sucrose, sweet; sodium chloride, salt; monosodium glutamate, umami) and a number of more complex flavors (almond, coffee, lemon, orange flower, peppermint, rose, vanilla). A pilot experiment was conducted on 6 participants in order to approximately match the subjective intensity of the 12 stimuli (see Table 1 for details of the concentrations and compositions of the stimuli used; all compounds were diluted in deionised water). Each sample consisted of $10 \mathrm{ml}$ of one of the solutions, presented in a 200-ml white plastic cup. The samples were identified by a number written on the cup.

The auditory stimuli came from an online musical instrument samples database from the University of Iowa Electronic Music Studios (http://theremin.music.uiowa.edu/MIS.html, retrieved October 31, 2009). They consisted of notes played by four types of instruments (piano, strings, woodwind, and brass). The pitch of the notes ranged from C2 $(64.4 \mathrm{~Hz})$ to $\mathrm{C} 6(1046.5 \mathrm{~Hz})$ in intervals of two tones. Thus, the participants had a choice of 52 different sounds ( 13 notes $\times 4$ instruments). The sounds were edited to last for $1,500 \mathrm{msec}$ and were presented over closed-ear headphones (Beyerdynamic DT 531) at a loudness of $70 \mathrm{~dB}( \pm 1 \mathrm{~dB})$. A subset of 16 notes $\left(\mathrm{C} 2, \mathrm{E} 3, \mathrm{G}_{\sharp} 4\right.$, and $\mathrm{C} 6$, played by the four types of instruments) was used in the second session.

\section{Procedure}

The instructions and choice of sounds were programmed in E-Prime. They were presented with a $60-\mathrm{Hz}$ refresh rate on an LCD monitor. Each trial started with instructions to the participants to rinse their mouths with tap water. This instruction remained on the screen for $15 \mathrm{sec}$ to ensure a minimum intertrial interval. The participants were then given the number of the sample that they were to taste and were reminded to take the whole sample $(10 \mathrm{ml})$ into their mouths, keep it in there for a few seconds, and then to spit it back into the cup. The participants then had to choose a sound to match the taste. The sounds were presented on four scales corresponding to the four types of instruments. Pitch increased from left to right. The sounds could be heard by clicking on the scales. The participants were free to click on as many of the sounds as they wished before making their choice. Note that on three occasions, a participant failed to pick a sound for a given taste/flavor. After having chosen, they rated the pleasantness, intensity, complexity, sweetness, sourness, bitterness, and saltiness of the taste/flavor on a separate piece of paper, using linear scales. The pleasantness scale was anchored by dislike extremely, indifference, and like extremely. The complexity scale was anchored by none and extremely complex. The other scales were anchored by none and extremely strong. The participants were also asked whether they had tasted the same taste before and could answer yes, no, or unsure. Finally, the participants were asked to identify the taste if possible. The 12 gustatory stimuli were presented twice in a random order, so that each participant completed a total of 24 trials.

In a second session, half of the participants rated a subset of the sounds on the pleasantness, intensity, and complexity scales. They were also asked to identify the instrument playing if possible.

\section{RESULTS}

\section{Consistency}

When presented with the same taste on the second occasion, the participants matched it to exactly the same sound as the first time in $11.3 \%$ of the cases. This value is significantly different from what would be expected if the participants had simply made a random choice among the 52 possible sounds $(1 / 52=1.9 \%)[t(407)=5.965$, $p<.01]$. This consistency in the pattern of the participants' responding was not driven by either pitch or instrument type alone. Both the same pitch and the same type of instrument were matched to a taste presented for the second time significantly more often than would be expected by chance, respectively in $21.8 \%$ (chance level: $7.7 \%)[t(407)=6.899, p<.01]$ and in $41.2 \%$ of the cases (chance level: $25 \%$ ) $[t(407)=6.631, p<.01]$.

\section{Pitch}

A repeated measures ANOVA, with GreenhouseGeisser correction, was conducted in order to assess whether there were any differences among the average pitches matched to the 12 tastes/flavors. The results indicated that the participants matched the 12 tastes/flavors to

Iable 1

Concentration and Composition of the Taste/Flavor Stimuli

\begin{tabular}{|c|c|c|c|}
\hline Stimuli & Origin & Composition & $\begin{array}{c}\text { Concentration } \\
\text { (\% Weight) }\end{array}$ \\
\hline Caffeine & Sigma-Aldrich & $\mathrm{C}_{8} \mathrm{H}_{10} \mathrm{~N}_{4} \mathrm{O}_{2}$ & 0.15 \\
\hline Citric acid & BDH laboratory supplies & $\mathrm{C}_{6} \mathrm{H}_{8} \mathrm{O}_{7} \cdot \mathrm{H}_{2} \mathrm{O}$ & 0.10 \\
\hline Sucrose & Silverspoon & $\mathrm{C}_{12} \mathrm{H}_{22} \mathrm{O}_{11}$ & 8.00 \\
\hline Salt & Saxa & $\mathrm{NaCl}$, sodium hexacyanoferrate II & 0.45 \\
\hline MSG & Sigma-Aldrich & $\mathrm{C}_{8} \mathrm{H}_{8} \mathrm{NNaO}_{4} \cdot \times \mathrm{H}_{2} \mathrm{O}$ & 2.00 \\
\hline Almond & Silverspoon & Sunflower oil, extract of almond & 2.00 \\
\hline Coffee & Marks \& Spencer & Water, alcohol (35\%), coffee extracts $(25 \%)$ & 1.00 \\
\hline Lemon & Marks \& Spencer & Ethyl alcohol $(85 \%)$, oil of lemon $(10 \%)$, water & 2.00 \\
\hline Orange flower & Marks \& Spencer & Propylene glycol-ethylalcohol $(40 \%)$, orange oil $(1.5 \%)$, natural flavors & 2.00 \\
\hline Peppermint & Silverspoon & Ethanol, water, flavoring & 0.25 \\
\hline Rose & Marks \& Spencer & Propylene glycol, alcohol (40\%), rose oil ( $2 \%)$, natural flavors & 1.50 \\
\hline Vanilla & Silverspoon & Water, ethanol, extract of vanilla & 10.00 \\
\hline
\end{tabular}




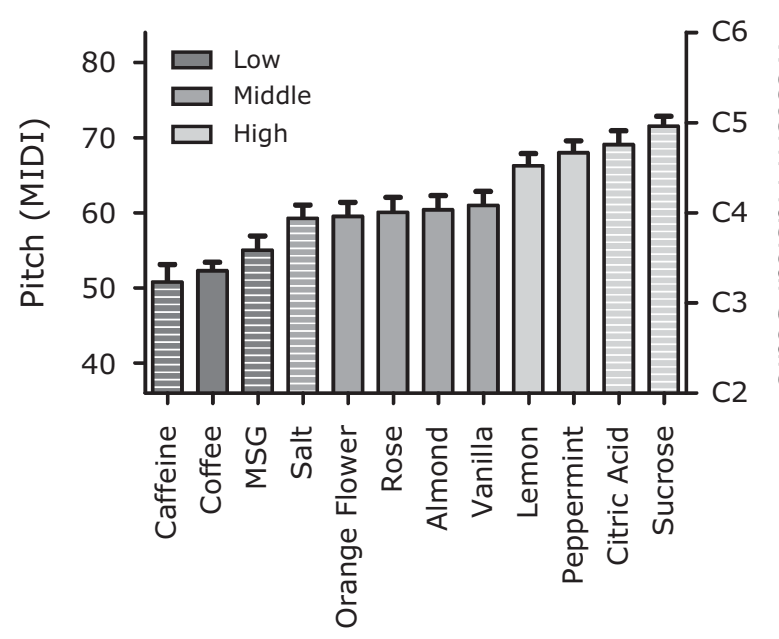

Figure 1. Mean pitch matched to each taste/flavor. MIDI (musical instrument digital interface) note numbers were used to code the pitch of the chosen notes. Western musical scale notation is shown on the right-hand $y$-axis. Basic tastes are depicted with lined bars. Tastes/flavors are grouped into low (dark gray), middle (gray), or high (light gray) groups according to the pitch that they were matched to.

pitch differently $[F(7.51,247.68)=16.51, p<.001]$. Following post hoc $t$ tests (Bonferroni-corrected), the tastes/ flavors were assigned to three groups according to the pitch that they were matched to (low, middle, or high; see Figure 1), so that the tastes/flavors within each group were not significantly different from each other. Moreover, each taste/flavor in the low-pitch group was significantly different from each taste/flavor in the high-pitch group.

\section{Types of Instruments}

A chi-square test for independence was conducted to assess whether different types of instruments were chosen for different tastes (see Figure 2). The results indicated that the tastes/flavors influenced the choice of instruments $\left[\chi^{2}(33, N=813)=157.27, p<.01\right]$. The strength of this effect, measured by computing Cramer's $V$, can be classified as medium ( $V=.25$ ) according to Cohen's (1988) guidelines. Further chi-square tests for goodness of fit were conducted to determine which tastes/flavors induced a distribution of instrument choice that was different from that expected by chance. Out of the 12 tastes/ flavors used, 7 gave rise to significant preferences in the choice of instrument (see Figure 2): caffeine $\left[\chi^{2}(3,68)=\right.$ $32.59, p<.01]$, sucrose $\left[\chi^{2}(3,68)=30.35, p<.01\right]$, coffee $\left[\chi^{2}(3,67)=19.39, p<.01\right]$, citric acid $\left[\chi^{2}(3,68)=\right.$ $17.53, p<.01]$, peppermint $\left[\chi^{2}(3,68)=13.65, p<\right.$ $.01]$, orange flower $\left[\chi^{2}(3,68)=10.00, p<.05\right]$, and salt $\left[\chi^{2}(3,68)=9.77, p<.05\right]$.

\section{Ratings of Tastes/Flavors}

The majority of the tastes/flavors (10 out of 12) were rated as unpleasant (i.e., the participants responded using the lower half of the scale, $<50 \%$ ), the two pleasant stimuli being peppermint $(M=57.6 \%, S D=12.7)$ and sucrose $(M=69.4 \%, S D=14.0)$. A chi-square test for independence was conducted to assess whether different types of

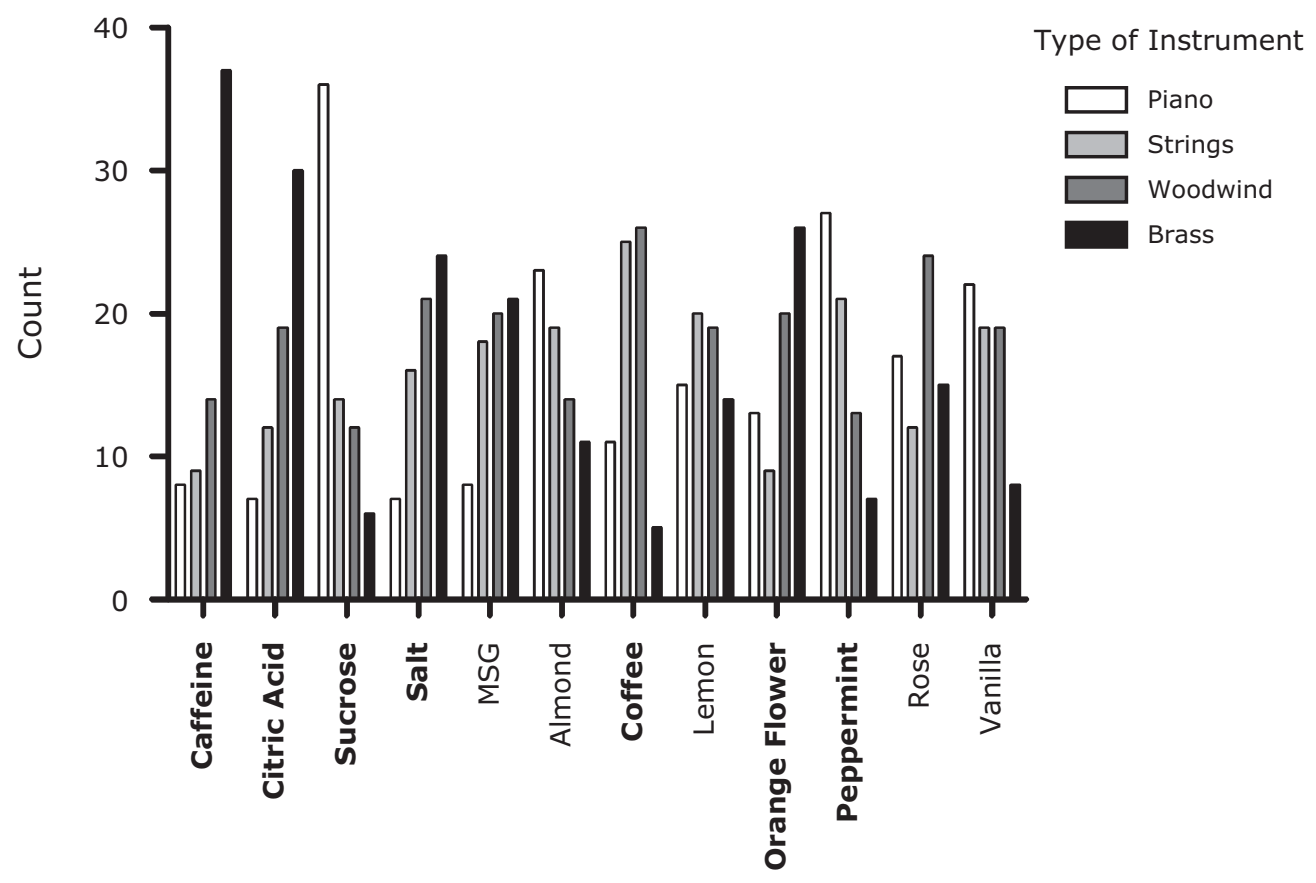

Taste/Flavor

Figure 2. Instruments matched to the tastes/flavors. Maximum count is 68 (34 participants, two trials for each stimulus). Stimuli for which there were significant preferences in the choice of instrument are in boldface. 
A

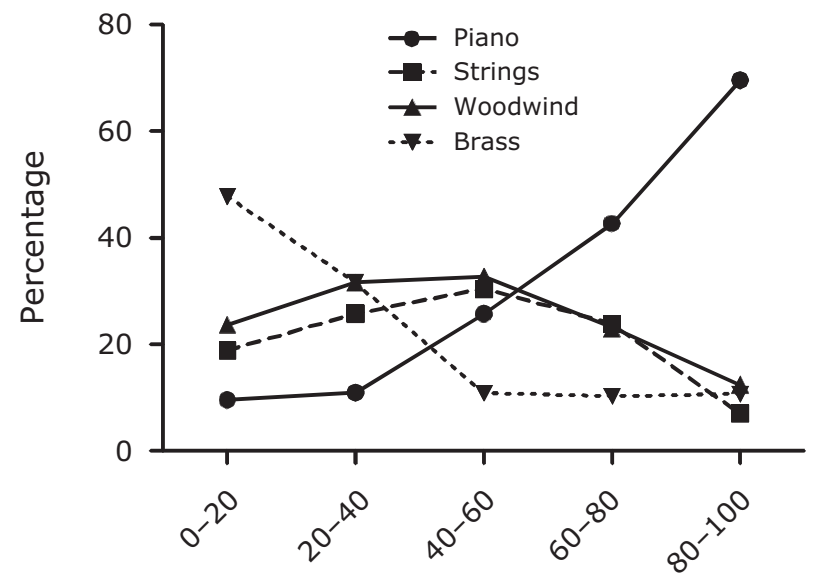

Pleasantness Rating (\%)

\section{B}

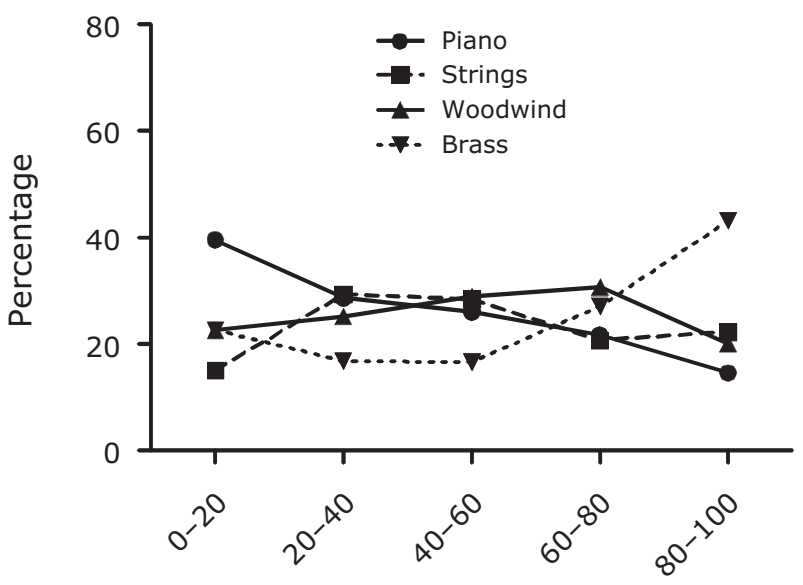

Intensity Rating (\%)

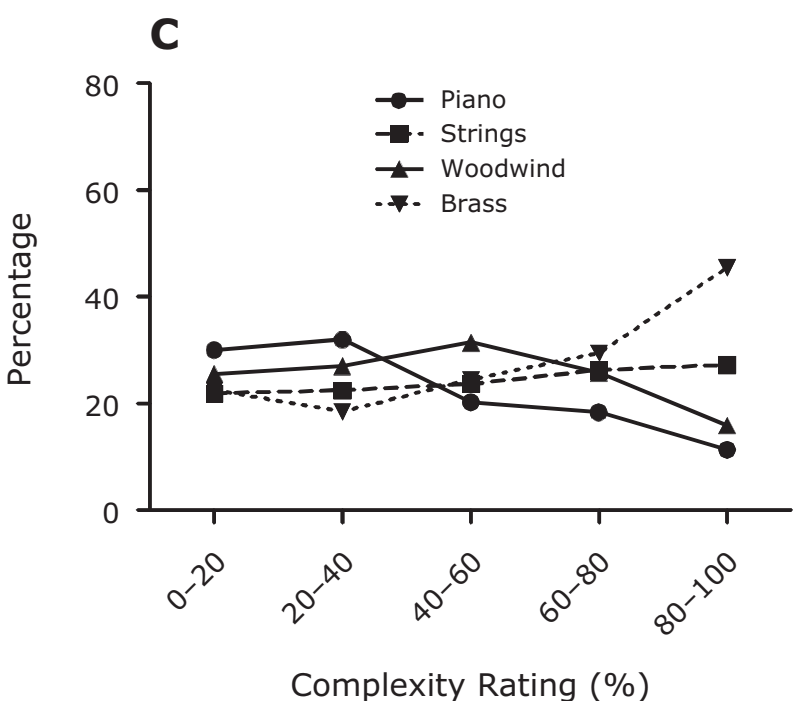

Figure 3. Choice of instrument (percentage of each instrument type per range group) in function of the pleasantness (A), intensity (B), and complexity (C) ratings. instruments were chosen for different pleasantness ratings (binned into five groups). The results indicated that the choice of instrument was not independent of the pleasantness rating $\left[\chi^{2}(12,812)=205.97, p<.01\right]$. The strength of this effect, once again measured by computing Cramer's $V$, can be classified as medium to large $(V=.29)$ according to Cohen's (1988) guidelines. Brass was often matched to unpleasant stimuli, whereas the piano was preferred for pleasant tastes/flavors (see Figure 3A).

A repeated measures ANOVA, with Greenhouse-Geisser correction, was conducted to determine whether there were any differences among the average intensities attributed to the 12 tastes/flavors. Even though a pilot experiment had been conducted to reduce differences in subjective intensity among the stimuli, the results indicated that the participants evaluated the intensity of the 12 tastes/flavors somewhat differently $[F(6.75,222.75)=5.36, p<.001]$. The results indicated that the choice of instrument was not independent of the intensity rating $\left[\chi^{2}(12,813)=50.1, p<.01\right]$ (see Figure $3 \mathrm{~B}$ ). However, the strength of this effect, measured by computing Cramer's $V$, can be classified as small ( $V=$ .14) according to Cohen's (1988) guidelines.

The choice of instrument was not independent of the complexity rating either $\left[\chi^{2}(12,813)=31.35, p<.01\right]$ (see Figure 3C). However, the strength of this effect, measured by computing Cramer's $V(V=.11)$, was small.

Basic tastes ratings were not independent of the choice of instrument, as assessed by chi-square tests (see Table 2 and Figures 4A-4D). The effect (measured with Cramer's $V$; see Table 2) was medium sized for the sweetness and bitterness ratings, whereas it was small for the sourness and saltiness ratings.

A one-way ANOVA revealed that both familiarity with the stimulus and identification of the taste/flavor influenced the choice of pitch $[F(2,804)=5.23, p<.01$, and $F(2,810)=24.72, p<.001$, respectively] (see Figures 5A and $5 \mathrm{~B}$ ). Familiarity with the stimulus and identification of the taste/flavor also had an effect on the choice of instrument $\left[\chi^{2}(6,805)=47.00, p<.01\right.$, and $\chi^{2}(6,813)=62.47$, $p<.01$, respectively] (see Figures 6A and 6B). Both effects were of medium size ( $V=.17$ and .20 , respectively).

\section{Principal Components Analysis}

The pitch and the seven other ratings (pleasantness, intensity, complexity, sweetness, sourness, bitterness, and saltiness) were subjected to a principal components analysis (PCA) using SPSS Version 16. The suitability of this approach was assessed first. The correlation matrix revealed the presence of several coefficients above .3. The Kaiser-Meyer-Oklin value was .607, attaining the recommended value of .6 (Kaiser, 1970, 1974), and Bartlett's test of sphericity (Bartlett, 1954) reached statistical significance, supporting the factorability of the correlation matrix. PCA revealed the presence of three components with eigenvalues over 1 , explaining $30.2 \%, 20.3 \%$, and $13.0 \%$ of the variance, respectively. We decided to keep only two components on the basis of an inspection of the screeplot, which showed a clear break after the second component, and on the basis of the results of parallel analysis, which showed only two components with eigen- 
I'able 2

Dependence of Basic Taste Ratings and Choice of Instruments Assessed by Chi-Square Tests and Cramer's $V$

\begin{tabular}{lccc}
\hline \multicolumn{1}{c}{ Taste } & $n$ & $\chi^{2}(12)$ & Cramer's $V$ \\
\hline Bitterness & 813 & 82.35 & .18 \\
Saltiness & 812 & 31.28 & .11 \\
Sourness & 813 & 53.74 & .15 \\
Sweetness & 813 & 78.90 & .18 \\
\hline
\end{tabular}

values exceeding the corresponding criterion values for a randomly generated data matrix of the same size ( 8 variables by 408 trials). Varimax rotation was performed. All variables loaded substantially onto only one component, except for bitterness, and, to a lesser extent, pleasantness, which loaded onto the two components (see Figure 7). The first component contributed $27.1 \%$ of the total variance explained of $50.5 \%$, whereas the second component contributed $23.4 \%$.

A

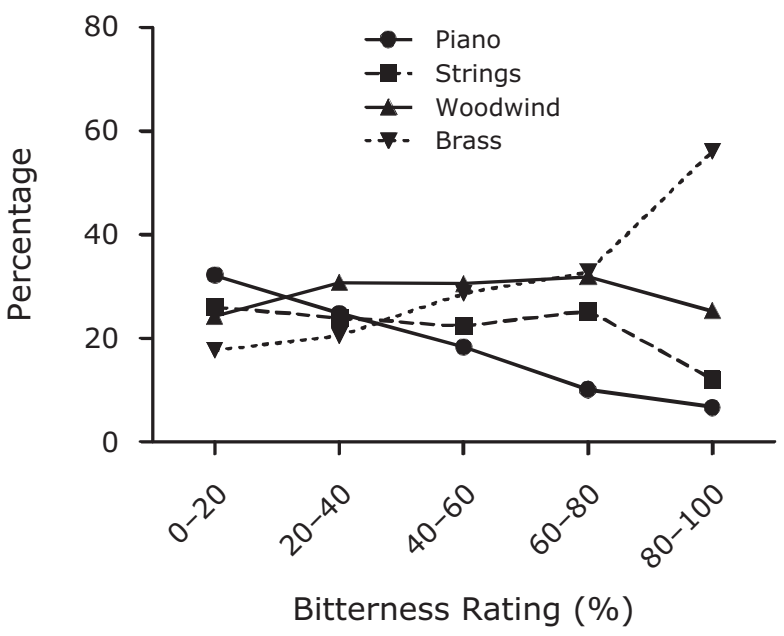

C

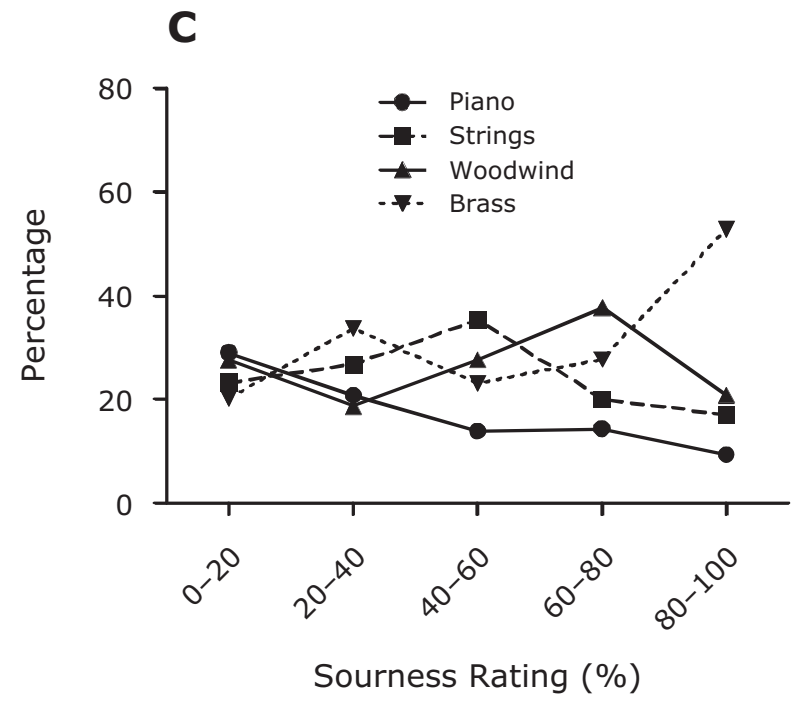

Sourness Rating (\%)

\section{Ratings of Sounds}

A two-way repeated measures ANOVA, with Greenhouse-Geisser correction, was conducted to assess the effect of the pitch and the instrument playing the sound on the ratings of pleasantness, intensity, and complexity. The results indicated a significant main effect of the type of instrument on pleasantness ratings $[F(2.69,43.05)=$ 65.87, $p<.001]$ and a significant interaction between type of instrument and pitch $[F(5.95,95.15)=7.58, p<$ $.001]$ (see Figure 8A). Intensity ratings were influenced by both the pitch and type of instrument $[F(2.27,34.29)=$ $11.22, p<.001$ and $F(2.33,37.32)=15.83, p<.001$, respectively] as well as by the interaction between these two factors $[F(6.31,101.01)=5.97, p<.001]$ (see Figure $8 \mathrm{~B})$. The only significant main effect on the complexity ratings was found for pitch $[F(2.30,36.76)=12.45$, $p<.001]$ (see Figure 8C). The instrument playing was correctly identified in $33.1 \%$ of the cases, and in a further

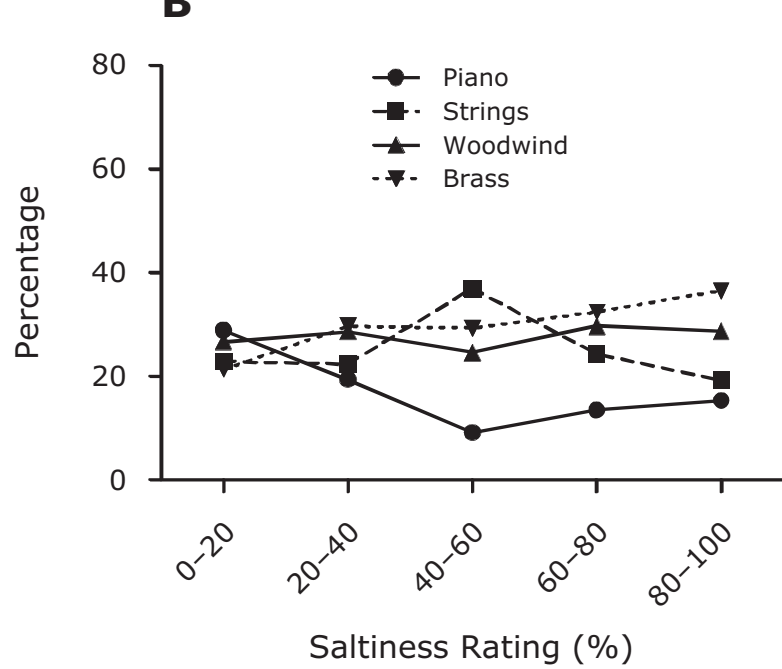

Figure 4. Choice of instrument (percentage of each instrument type per range group) in function of the bitterness (A), saltiness (B), sourness (C), and sweetness (D) ratings. 


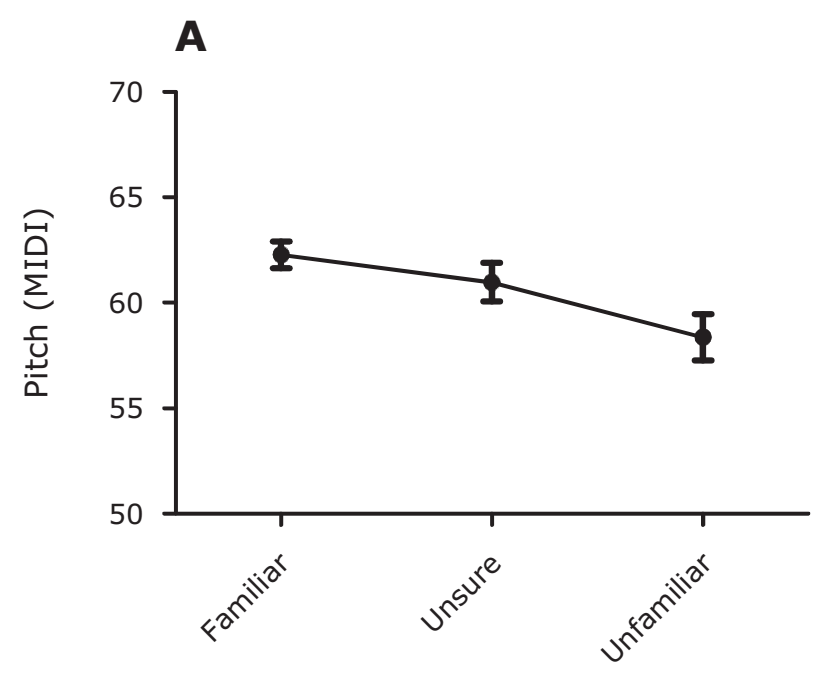

Taste Familiarity

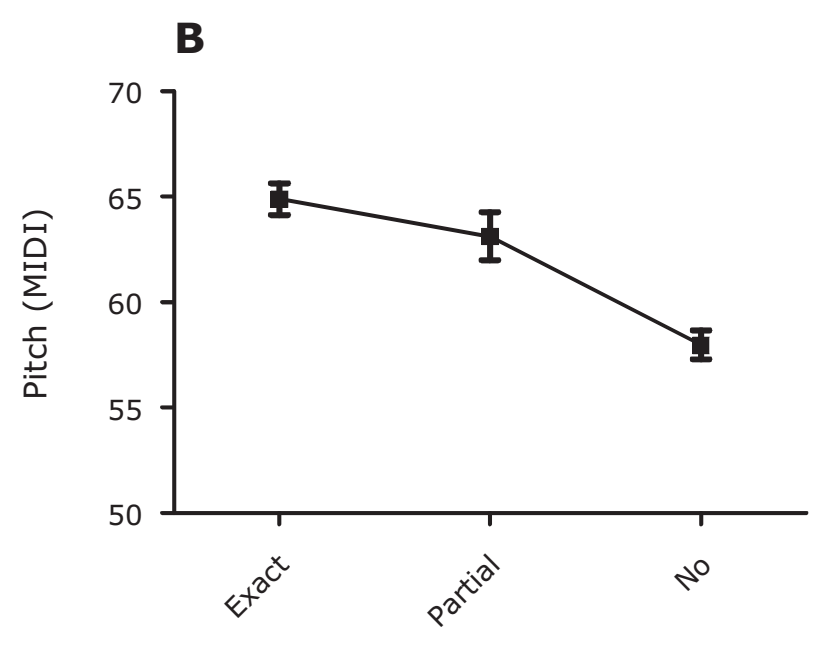

Taste Identification

Figure 5. Choice of pitch as a function of the familiarity (A) and identification of the taste/flavor (B). Error bars represent 1 SEM.

$27.6 \%$, the type of instrument (e.g., strings) was identified. The piano was recognized in $100 \%$ of the cases, whereas brass instruments were only identified exactly in $4.4 \%$ of the cases (and in a further $48.5 \%$, the participants recognized that it was a wind instrument). Strings and woodwinds reached intermediate scores (respectively $14.7 \%$ and $13.2 \%$ of correct identification).

\section{DISCUSSION}

The consistency in the choice of the sounds matched to each taste/flavor by the participants in the present study confirms the fact that they did not choose randomly. It is unlikely that the consistency observed was due to a memory effect (i.e., the participants trying to be consistent by remembering their first choice), since most of them were unaware that the same stimuli were presented twice. Both the pitch of the sound and the instrument playing it influenced the participants' choice.

The choice of the pitch attributed to the basic tastes confirms the results obtained previously by Crisinel and Spence $(2009,2010)$, in which the names of food or drink items were used instead of actual tastes/flavors. Namely, the present results also show that sour and sweet tastes are preferentially associated with high-pitched sounds. There is also some evidence of a bitter-low-pitch association, which was first observed (compared with sour taste) by Crisinel and Spence (2009) before being discarded when the associations were investigated separately (Crisinel \& Spence, 2010). This association might have been overlooked previously because of the choice of stimuli used to represent the bitter taste in our earlier study (beer, cof-
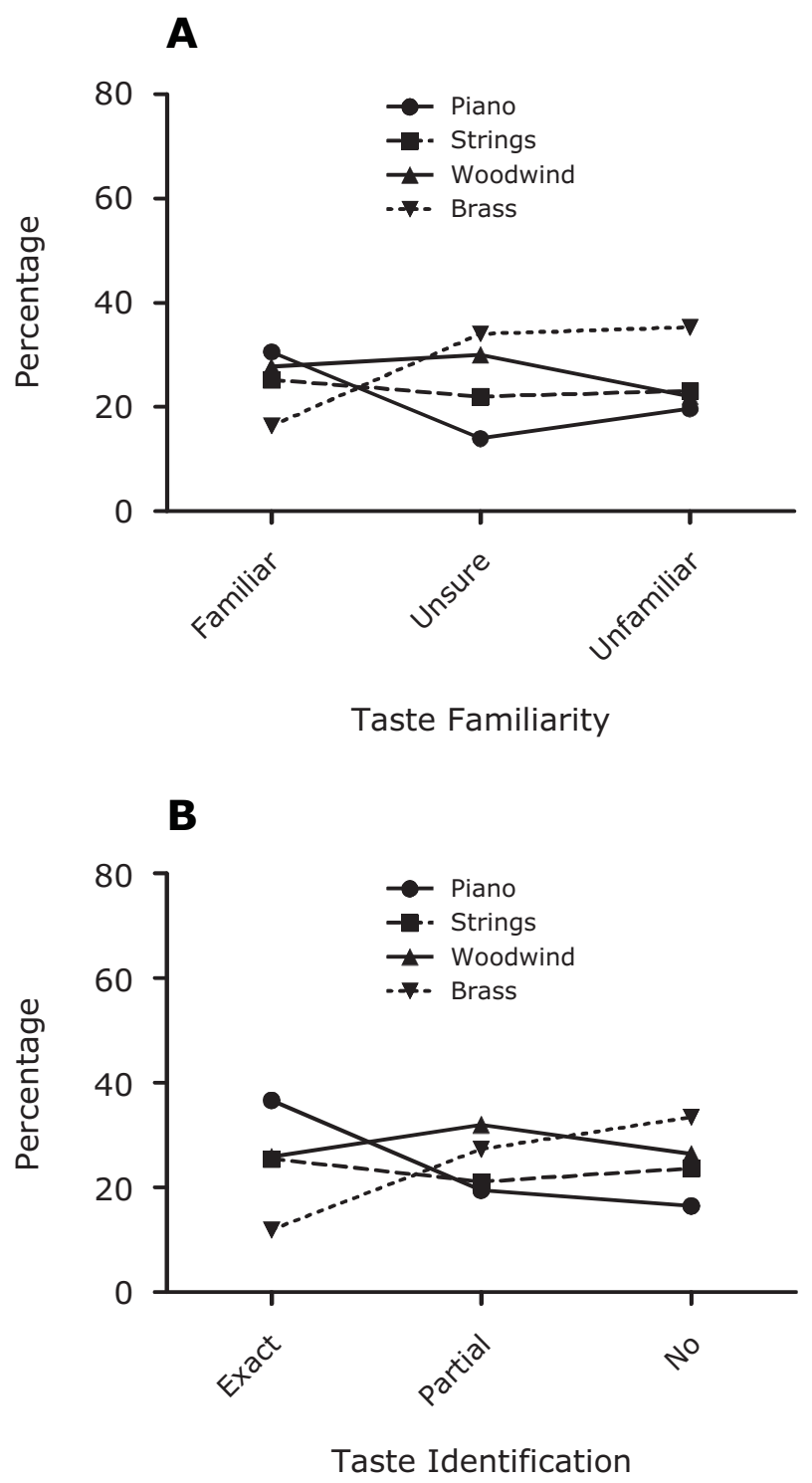

Figure 6. Choice of instrument (percentage of each instrument type per response) in function of the familiarity (A) and identification of the taste/flavor (B). 


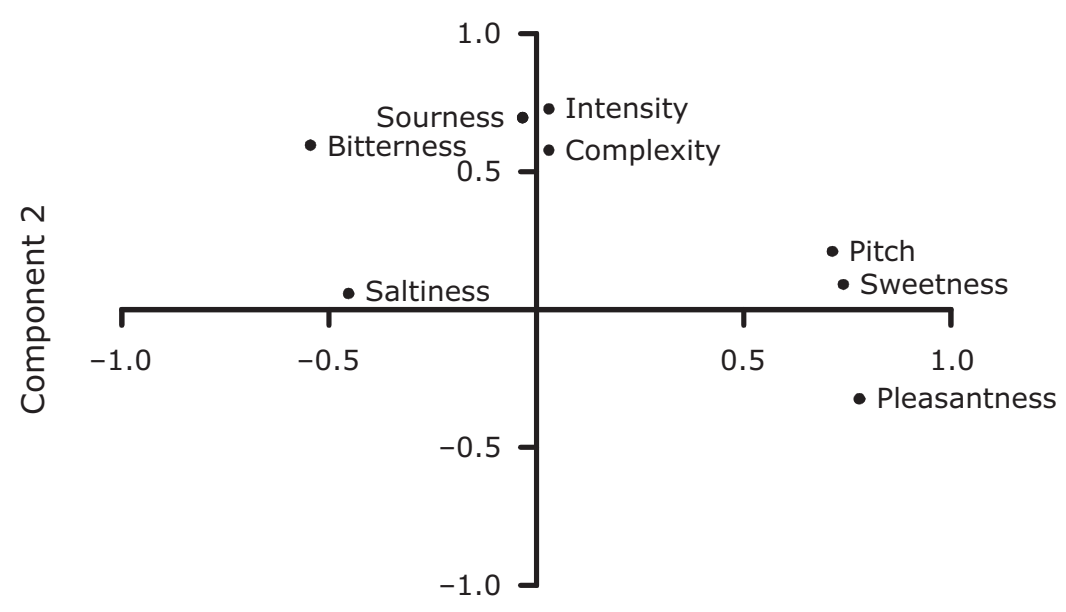

Component 1

Figure 7. Loadings of the pitch and the various ratings on the rotated components extracted through principal components analysis.

fee, dark chocolate, and tonic water), which the participants did not agree on unanimously. The absence of an association between salty tastes and extreme pitch is also confirmed by our results (at least at the salt intensity used here). Moreover, our results suggested a possible association between umami and low pitch.

Note that the strongest associations with pitch in the present experiment were found for basic tastes, rather than for flavors. Four out of seven flavors were classified in the middle group. The presence of the lemon flavor in the highpitch group might be due to its high sourness rating $(M=$ $40.6 \%, S D=27.6$, second after citric acid). The coffee flavor (unsweetened) is traditionally thought of as tasting bitter, which might explain its classification in the lowpitch group. However, in this case, the explanation is less convincing than for lemon, since both orange flower and rose flavors were rated as more bitter $(M=42.3 \%, S D=$ 27.2 , and $M=40.6 \%, S D=25.5$, respectively) than the coffee flavor $(M=38.7 \%, S D=25.7)$. These weaker associations between flavors and pitch are surprising, given that odors have been shown to have associations with pitch (Belkin, Martin, Kemp, \& Gilbert, 1997). However, several differences between Belkin et al.'s study and the present experiment might help to explain the contradictory results: Most of the odors used by Belkin et al. were flower or plant odors, rather than food odors; the odors were smelled orthonasally, in contrast to the present study, in which odors could be smelled both ortho- and retronasally; and the attention of the participants was focused on the odors, rather than on the tastes, in Belkin et al.'s study.

The classification of the peppermint flavor in the high-pitch group suggests a possible association between trigeminal stimulation and high pitch. It would be interesting to explore other kinds of trigeminal stimuli (e.g., chili, horseradish) in order to determine whether high pitch is associated with trigeminal stimulation more generally or only with the perceived coolness of peppermint.

Sounds played by the piano were preferentially chosen for tastes/flavors rated as pleasant, whereas unpleasant tastes were matched most often to sounds played by brass instruments (see Figure 3A). Brass instruments were also preferred for stimuli rated as tasting very bitter or sour (between $80 \%$ and $100 \%$; see Figures $4 \mathrm{~A}$ and $4 \mathrm{C}$ ). This tendency corresponds to the pleasantness ratings of the sounds (see Figure 8A): The piano was rated as the most pleasant at all pitches, and the brass instruments as the most unpleasant, especially at lower pitches. This suggests that the choice of the instrument matched to a taste can largely be attributed to a matching of the hedonic values of the two stimuli. However, high saltiness ratings did not elicit such a strong preference for brass instruments (see Figure 4B), even though sodium chloride was rated as unpleasant $(M=32.0 \%, S D=19.1)$.

Tastes that the participants reported being familiar with or that they could correctly identify were matched to higher pitched sounds (see Figures 5A and 5B). Furthermore, sounds played by the piano were preferred for these tastes; whereas brass instruments were chosen more often for unfamiliar tastes (see Figures 6A and 6B). The preference in the type of instrument for familiar tastes parallels the recognition of the instruments themselves, since the piano was the most easily recognized (on 100\% of trials). Moreover, the choices made for familiar tastes/flavors were very similar to the choices for pleasant tastes, as would be expected as a result of the well-known association between familiarity and liking (Bornstein, 1989; Maslow, 1937; Monahan, Murphy, \& Zajonc, 2000; Suzuki \& Gyoba, 2008).

PCA suggests two components to account for the various ratings of the tastes, as well as for the pitch matched to the tastes (see Figure 7). The first component has a strong loading of the intensity factor, sourness, bitterness, and complexity. The second component has strong loadings of pleasantness and sweetness. The choice of pitch seems related to this component. These two components might correspond to the first two (potency and evaluation) of the dimensions used in the semantic differential scale (Osgood, 1952) that has been applied to a variety of stimuli and recently to odors (Dalton, Maute, Oshida, Hikichi, 


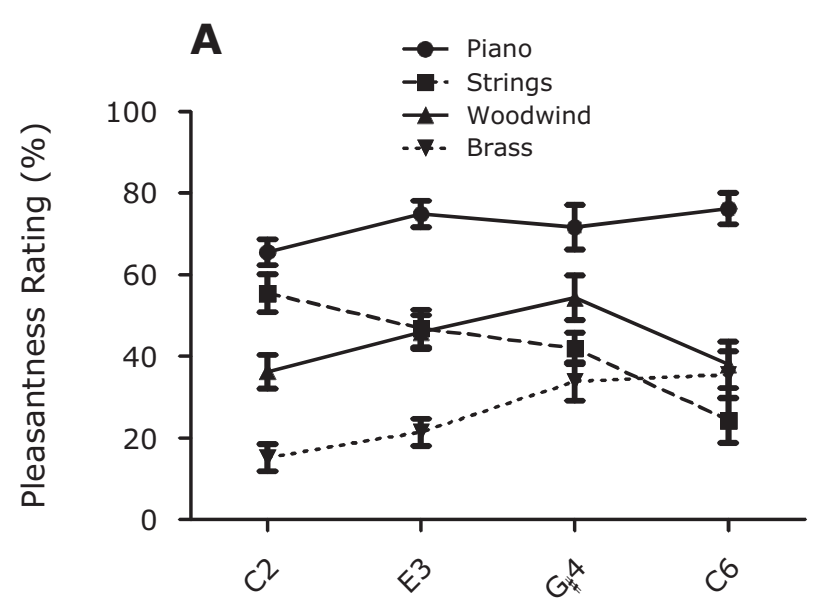

Pitch

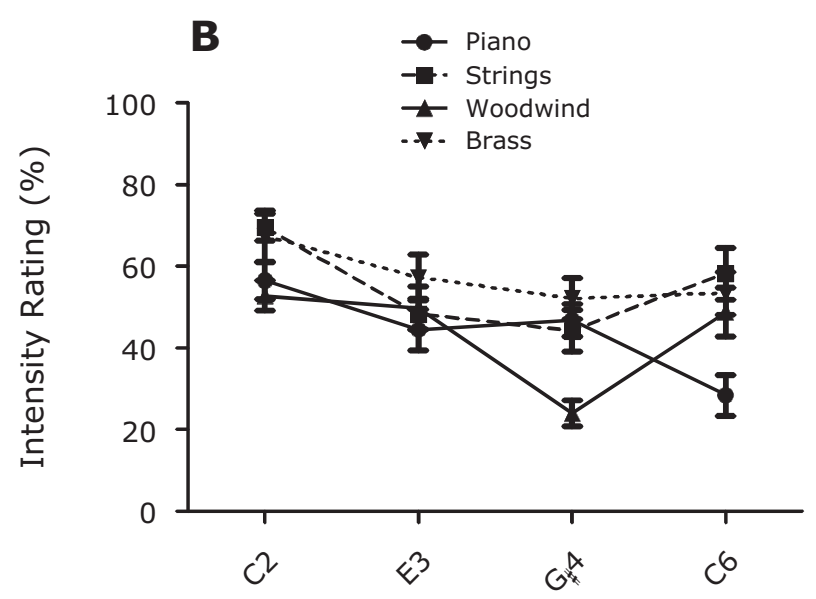

Pitch

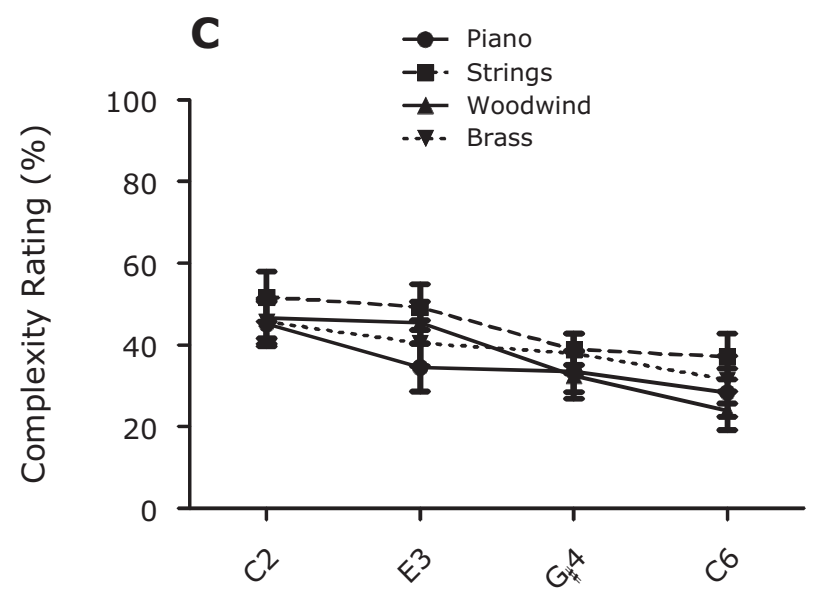

Pitch

Figure 8. Pleasantness (A), intensity (B), and complexity (C) ratings of a subset of the sounds used in the experiment.
\& Izumi, 2008). The third dimension, activity, might be linked to the time course of the perception of taste, which is itself influenced by parameters that were not varied in our experiment, such as the texture of the stimuli.

Given that similar results were also obtained when tastes were evoked by the names of food items (Crisinel $\&$ Spence, 2009, 2010), the associations must occur at a higher cognitive level, as was suggested by Martino and Marks's (1999) semantic-coding hypothesis.

A deeper knowledge of the nature of the associations between sounds and tastes/flavors would be very useful for the marketing of food products (see Klink, 2000, 2001). One might, for example, ask whether Sweet'N Low was necessarily the best way to name an artificial sweetener. Our results would certainly suggest otherwise. If single sounds can be matched to tastes/flavors, it seems likely that a similar matching would occur with music and/or complex combinations of environmental sounds. Should this hypothesis be confirmed by subsequent research, there would be some intriguing implications for the design of dining areas and restaurants, so that environmental sounds and/or background music can be better matched to the dishes that are consumed there (see Spence, Shankar, \& Blumenthal, 2010). It would also have important consequences for the marketing of various food products, in terms of the music used in advertisements, the sounds made by packaging (Spence \& Zampini, 2006), and background music in shops (North \& Hargreaves, 2008; Spence \& Shankar, 2010).

Although further research might help better understand the dimensions underlying the cross-modal correspondences observed here, they seem to require a complex model, rather than being a simple matching of obvious physical properties or of evaluation (although it plays a role, as was suggested by the PCA analysis). Whatever the underlying mechanism of these auditory-gustatory associations is, they add to the growing list of cross-modal correspondences, which raises another question: Why do we associate stimuli from different sensory modalities so easily? One hypothesis that has been put forward is that we need to simplify the constant flow of sensory information to make sense of the complex world around us. To that end, it is useful to associate attributes from different sensory modalities, such as the color of a fruit to its taste, since it indicates its ripeness (see Spence, Levitan, Shankar, \& Zampini, 2010), or a deep, low-pitched growl to a large animal and a high-pitched squeak to a small one (see Gallace \& Spence, 2006). We might have evolved to easily compare and combine information from different sensory modalities as a way to make better predictions when we only have partial information. As a side effect, we would tend to associate attributes even when they have no predictive value. Seeing synesthesia in this light makes it appear a much less surprising condition than is usually the case. It would then be the result of a mechanism present in all of us, but pushed to the extreme. Describing cross-modal correspondences as weak synesthesia (Martino \& Marks, 2001) goes in that direction. This view would also fit with the hypothesis that synesthesia may be attributable to a lack of inhibition in otherwise normal neural pathways (see Grossenbacher \& Lovelace, 2001). 
In conclusion, our results raise interesting questions about the important dimensions in our representation of tastes and flavors and how we tend to match them to dimensions in other sensory modalities. They also open the way for potentially important applications in the design and marketing of food products.

\section{AUTHOR NOTE}

A.-S. Crisinel thanks the Berrow Foundation for funding her research. Correspondence concerning this article should be addressed to A.-S. Crisinel, Department of Experimental Psychology, University of Oxford, South Parks Road, Oxford, OX1 3UD, UK (e-mail: anne-sylvie .crisinel@psy.ox.ac.uk).

\section{REFERENCES}

Auvray, M., \& Spence, C. (2008). The multisensory perception of flavor. Consciousness \& Cognition, 17, 1016-1031.

BARTLETT, M. S. (1954). A note on multiplying factors for various chi square approximations. Journal of the Royal Statistical Society, 16B, 296-298.

Belkin, K., Martin, R., Kemp, S. E., \& Gilbert, A. N. (1997). Auditory pitch as a perceptual analogue to odor quality. Psychological Science, 8, 340-342.

BorNSTEIN, R. F. (1989). Exposure and affect: Overview and meta-analysis of research, 1968-1987. Psychological Bulletin, 106, 265-289.

Calvert, G. A., Spence, C., \& Stein, B. E. (Eds.) (2004). The handbook of multisensory processes. Cambridge, MA: MIT Press.

CoHEn, J. (1988). Statistical power analysis for the behavioral sciences. Hillsdale, NJ: Erlbaum.

Crisinel, A.-S., \& Spence, C. (2009). Implicit association between basic tastes and pitch. Neuroscience Letters, 464, 39-42.

CRISINEL, A.-S., \& SPENCE, C. (2010). A sweet sound? Food names reveal implicit associations between taste and pitch. Perception, 39, 417-425.

Cytowic, R. E., \& EAGLeman, D. M. (2009). Wednesday is indigo blue: Discovering the brain of synesthesia. Cambridge, MA: MIT Press.

Dalton, P., Maute, C., Oshida, A., Hikichi, S., \& Izumi, Y. (2008). The use of semantic differential scaling to define the multidimensional representation of odors. Journal of Sensory Studies, 23, 485-497.

Delwiche, J. (1996). Are there 'basic' tastes? Trends in Food Science \& Technology, 7, 411-415.

Djordjevic, J., Zatorre, R. J., \& Jones-Gotman, M. (2004). Effects of perceived and imagined odors on taste detection. Chemical Senses, 29, 199-208

ERICKSON, R. P. (2008). A study of the science of taste: On the origins and influence of the core ideas. Behavioral \& Brain Sciences, 31, 59-75.

Evans, K. K., \& Treisman, A. (2010). Natural cross-modal mappings between visual and auditory features. Journal of Vision, 10(Art. 6), 1-12.

Gallace, A., \& Spence, C. (2006). Multisensory synesthetic interactions in the speeded classification of visual size. Perception \& Psychophysics, 68, 1191-1203.

GaLton, F. (1880). Visualised numerals. Nature, 22, 494-495.

Grossenbacher, P. G., \& Lovelace, C. T. (2001). Mechanisms of synesthesia: Cognitive and physiological constraints. Trends in Cognitive Sciences, 5, 36-41.

HARRISON, J. (2001). Synaesthesia: The strangest thing. Oxford: Oxford University Press.

Hochel, M., \& MiLÁN, E. G. (2008). Synaesthesia: The existing state of affairs. Cognitive Neuropsychology, 25, 93-117.

Holt-Hansen, K. (1968). Taste and pitch. Perceptual \& Motor Skills, 27, 59-68.

Holt-Hansen, K. (1976). Extraordinary experiences during cross-modal perception. Perceptual \& Motor Skills, 43, 1023-1027.

JoHnson, A., JEPMA, M., \& DE JonG, R. (2007). Colours sometimes count: Awareness and bidirectionality in grapheme-colour synaesthesia. Quarterly Journal of Experimental Psychology, 60, 1406-1422.

KaIsER, H. (1970). A second generation Little Jiffy. Psychometrika, 35, 401-415.

KaIsER, H. (1974). An index of factorial simplicity. Psychometrika, 39, 31-36.
KLINK, R. R. (2000). Creating brand names with meaning: The use of sound symbolism. Marketing Letters, 11, 5-20.

KLINK, R. R. (2001). Creating meaningful new brand names: A study of semantics and sound symbolism. Journal of Marketing: Theory \& Practice, 9, 27-34.

MARKs, L. E. (1982). Bright sneezes and dark coughs, loud sunlight and soft moonlight. Journal of Experimental Psychology: Human Perception \& Performance, 8, 177-193.

Martino, G., \& MarKs, L. E. (1999). Perceptual and linguistic interactions in speeded classification: Tests of the semantic coding hypothesis. Perception, 28, 903-923.

Martino, G., \& Marks, L. E. (2000). Cross-modal interaction between vision and touch: The role of synesthetic correspondence. Perception, 29, 745-754.

Martino, G., \& Marks, L. E. (2001). Synesthesia: Strong and weak. Current Directions in Psychological Science, 10, 61-65.

MasLow, A. H. (1937). The influence of familiarization on preference. Journal of Experimental Psychology, 21, 162-180.

Monahan, J. L., Murphy, S. T., \& Zajonc, R. B. (2000). Subliminal mere exposure: Specific, general, and diffuse effects. Psychological Science, 11, 462-466.

North, A., \& Hargreaves, D. (2008). The social and applied psychology of music. Oxford: Oxford University Press.

O’Mahony, M. (1983). Gustatory responses to nongustatory stimuli. Perception, 12, 627-633.

OsGood, C. E. (1952). The nature and measurement of meaning. Psychological Bulletin, 49, 197-237.

Parise, C. V., \& SPEnce, C. (2009). "When birds of a feather flock together": Synesthetic correspondences modulate audiovisual integration in non-synesthetes. PLOS ONE, 4(5), e5664.

SAGIV, N., \& WARD, J. (2006). Cross-modal interactions: Lessons from synesthesia. Progress in Brain Research, 155, 263-275.

Simner, J., Cuskley, C., \& Kirby, S. (2010). What sound does that taste? Crossmodal mappings across gestation and audition. Perception, 39, 553-569.

Small, D. M., \& Prescott, J. (2005). Odor/taste integration and the perception of flavor. Experimental Brain Research, 166, 345-357.

Spence, C., Levitan, C. A., Shankar, M. U., \& Zampini, M. (2010). Does food color influence taste and flavor perception in humans? Chemosensory Perception, 3, 68-84.

Spence, C., \& Shankar, M. U. (2010). The influence of auditory cues on the perception of, and responses to, food and drink. Journal of Sensory Studies, 25, 406-430.

Spence, C., Shankar, M. U., \& Blumenthal, H. (2010). "Sound bites": Auditory contributions to the perception and consumption of food and drink. In F. Bacci \& D. Mecher (Eds.), Art and the senses (pp. 207-238). Oxford: Oxford University Press.

SPEnce, C., \& Zampini, M. (2006). Auditory contributions to multisensory product perception. Acta Acustica United With Acustica, 92 , 1009-1025.

Stevenson, R. J., Boakes, R. A., \& Prescott, J. (1998). Changes in odor sweetness resulting from implicit learning of a simultaneous odor-sweetness association: An example of learned synesthesia. Learning \& Motivation, 29, 113-132.

STEVENSON, R. J., \& ToMicZeK, C. (2007). Olfactory-induced synesthesias: A review and model. Psychological Bulletin, 133, 294-309.

Suzuki, M., \& Gyoba, J. (2008). Visual and tactile cross-modal mere exposure effects. Cognition \& Emotion, 22, 147-154.

Ward, J., Huckstep, B., \& Tsakanikos, E. (2006). Sound-color synaesthesia: To what extent does it use cross-modal mechanisms common to us all? Cortex, 42, 264-280.

\section{NOTE}

1. The concept of basic tastes is a much debated issue (e.g., Delwiche, 1996; Erickson, 2008). It is certainly not our purpose to tackle this issue in the present study. We use the terms sweet, salty, sour, bitter, and umami as useful labels, no matter whether they represent basic tastes, points on a taste continuum, or something else.

(Manuscript received March 25, 2010; revision accepted for publication June 2, 2010.) 